\title{
POINTWISE ESTIMATES FOR THE BERGMAN KERNEL OF THE WEIGHTED FOCK SPACE
}

\author{
JORDI MARZO AND JOAQUIM ORTEGA-CERDÀ
}

\begin{abstract}
We prove upper pointwise estimates for the Bergman kernel of the weighted Fock space of entire functions in $L^{2}\left(e^{-2 \phi}\right)$ where $\phi$ is a subharmonic function with $\Delta \phi$ a doubling measure. We derive estimates for the canonical solution operator to the inhomogeneous CauchyRiemann equation and we characterize the compactness of this operator in terms of $\Delta \phi$.
\end{abstract}

\section{INTRODUCTION}

Let $\phi$ be a subharmonic function in $\mathbb{C}$ whose Laplacian $\Delta \phi$ is a doubling measure. For $1 \leq$ $p<\infty$, we consider the Fock spaces

$$
\mathcal{F}_{\phi}^{p}=\left\{f \in \mathcal{H}(\mathbb{C}):\|f\|_{\mathcal{F}_{\phi}^{p}}^{p}=\int_{\mathbb{C}}|f(z)|^{p} e^{-p \phi(z)} d m(z)<\infty\right\}
$$

and

$$
\mathcal{F}_{\phi}^{\infty}=\left\{f \in \mathcal{H}(\mathbb{C}):\|f\|_{\mathcal{F}_{\phi}^{\infty}}=\sup _{z \in \mathbb{C}}|f(z)| e^{-\phi(z)}<\infty\right\}
$$

where $d m$ denotes the Lebesgue measure in $\mathbb{C}$.

Let $\overline{K(z, \zeta)}=K_{z}(\zeta)$ denote the Bergman kernel for $\mathcal{F}_{\phi}^{2}$, i.e. for any $f \in \mathcal{F}_{\phi}^{2}$

$$
f(z)=\left\langle f, K_{z}\right\rangle_{\mathcal{F}_{\phi}^{2}}=\int_{\mathbb{C}} f(\zeta) K(z, \zeta) e^{-2 \phi(\zeta)} d m(\zeta), \quad z \in \mathbb{C} .
$$

If $\mu=\Delta \phi$, the function $\rho(z)$ denotes the positive radius such that $\mu(D(z, \rho(z)))=1$. The function $\rho^{-2}$ can be considered as a regularized version of $\Delta \phi$, see [Chr91] or [MMO03]. We write $D^{r}(z)=D(z, r \rho(z))$ and $D^{1}(z)=D(z)$ (we will write $\rho_{\phi}(z)$ and $D_{\phi}^{r}(z)$ if we need to stress the dependence on $\phi$ ).

In this context the Bergman kernel has already been studied. In [Chr91] M. Christ obtained pointwise estimates under the hypothesis that $\phi$ is a subharmonic function such that $\mu=\Delta \phi$ is a doubling measure and

$$
\inf _{z \in \mathbb{C}} \mu(D(z, 1))>0 .
$$

This result was extended to several complex variables by H. Delin and N. Lindholm in [Del98] and [Lin01] under similar hypothesis. They obtain a very fast decay of the Bergman kernel away from the diagonal.

Date: March 13, 2009.

Key words and phrases. Bergman kernel, Compactness canonical operator.

Supported by projects MTM2008-05561-C02-01 and 2005 SGR00611. 
We will remove hypothesis (1) (which in somes sense is related to the strict pseudoconvexity) and keep only the doubling condition (that is morally closer to finite-type). We still obtain some decay away from the diagonal, we derive estimates for the canonical solution operator to the inhomogeneous Cauchy-Riemann equation and we characterize the compactness of this operator in terms of $\Delta \phi$. Our main result is the following estimate.

Theorem 1.1. Let $K(z, \zeta)$ be the Bergman kernel for $\mathcal{F}_{\phi}^{2}$. There exist positive constants $C$ and $\epsilon$ (depending only on the doubling constant for $\Delta \phi$ ) such that for any $z, \zeta \in \mathbb{C}$

$$
|K(z, \zeta)| \leq C \frac{1}{\rho(z) \rho(\zeta)} \frac{e^{\phi(z)+\phi(\zeta)}}{\exp \left(\frac{|z-\zeta|}{\rho(z)}\right)^{\epsilon}} .
$$

Although the estimate above seems to be asymmetric in the variables $z, \zeta$ one can see that for $|z-\zeta|<C \max \{\rho(z), \rho(\zeta)\}$ the values of $\rho(z)$ and $\rho(\zeta)$ are comparable, see Lemma 2.3. Also when $|z-\zeta| \geq C \max \{\rho(z), \rho(\zeta)\}$ one can use Lemma 2.6 to see that the same estimate holds with $\rho(\zeta)$ inside the exponential for a different positive exponent $\epsilon$ (this new exponent depending only on the doubling constant for $\Delta \phi$ ). The symmetry becomes apparent when we write (2) in terms of the distance $d_{\phi}$ induced by the metric $\rho^{-2}(z) d z \otimes d \bar{z}$. Indeed, by using Lemma 2.6 one can write (2) as

$$
|K(z, \zeta)| \leq C \frac{1}{\rho(z) \rho(\zeta)} \frac{e^{\phi(z)+\phi(\zeta)}}{\exp \left(d_{\phi}(z, \zeta)^{\epsilon}\right)}
$$

for some $\epsilon>0$ (different from the previous one but still positive). The estimate proved in [Chr91] for the Bergman kernel of $\mathcal{F}_{\phi}^{2}$ defined for a $\phi$ with doubling Laplacian and satisfying (1) is

$$
|K(z, \zeta)| \leq C \frac{1}{\rho^{2}(z)} \frac{e^{\phi(z)+\phi(\zeta)}}{\exp \left(\epsilon d_{\phi}(z, \zeta)\right)},
$$

for some $\epsilon>0$ and all $z, \zeta \in \mathbb{C}$.

Let $N$ be the canonical solution operator to $\bar{\partial}$, i.e. $\bar{\partial} N f=f$ and $N f$ is of minimal $L^{2}\left(e^{-2 \phi}\right)$ norm and let $C(z, \zeta)$ be the integral kernel such that

$$
N f(z)=\int_{\mathbb{C}} e^{\phi(z)-\phi(\zeta)} C(z, \zeta) f(\zeta) d m(\zeta) .
$$

The boundedness and compactness of this canonical solution operator from $L^{2}\left(e^{-2 \phi}\right)$ to itself has been extensively studied in one and several variables; for a survey on this problem and its applications see [FS01]. It is shown in [Has06] that for weights on the class considered by M. Christ, the condition $\rho(z) \rightarrow 0$ when $|z| \rightarrow \infty$ is sufficient for compactness. In the same paper it is shown that the canonical solution operator with $\phi(z)=|z|^{2}$ fails to be compact, all these results are contained in Theorem 1.3 . Finally, in [HH07] the authors prove a result similar to Theorem 1.3 with some extra regularity conditions on $\Delta \phi$.

With Theorem 1.1 we obtain a pointwise estimate on the kernel of the canonical solution operator. 
Theorem 1.2. There exists an integral kernel $G(z, \zeta)$ such that

$$
u(z)=\int_{\mathbb{C}} e^{\phi(z)-\phi(\zeta)} G(z, \zeta) f(\zeta) d m(\zeta),
$$

solves $\bar{\partial} u=f$ and

$$
|G(z, \zeta)| \lesssim\left\{\begin{array}{cl}
|z-\zeta|^{-1}, & |z-\zeta| \leq \rho(z) \\
\rho^{-1}(z) \exp \left(-d_{\phi}(z, \zeta)^{\epsilon}\right), & |z-\zeta| \geq \rho(z)
\end{array}\right.
$$

Moreover, the integral kernel $C(z, \zeta)$ giving the canonical solution to $\bar{\partial}$ in $L^{2}\left(e^{-2 \phi}\right)$ has the same estimate (with a different exponent $\epsilon>0$ ).

One can compare this result with the estimate on [Chr91, Theorem 1.13] where the author proves that

$$
|C(z, \zeta)| \lesssim\left\{\begin{array}{cl}
|z-\zeta|^{-1}, & |z-\zeta| \leq \rho(z) \\
\rho^{-1}(z) \exp \left(-\epsilon d_{\phi}(z, \zeta)\right), & |z-\zeta| \geq \rho(z)
\end{array}\right.
$$

As an application of the estimate (2) we characterize the compactness of the canonical solution operator to $\bar{\partial}$ in terms of the measure $\Delta \phi$.

Theorem 1.3. Let $\phi$ be a subharmonic function such that $\Delta \phi$ is doubling. The canonical solution operator $N$ of minimal norm in $L^{2}\left(e^{-2 \phi}\right)$ to the inhomogeneous $\bar{\partial}$-equation defines a bounded compact operator from $L^{2}\left(e^{-2 \phi}\right)$ to itself if and only if $\rho(z) \rightarrow 0$ when $|z| \rightarrow \infty$.

Any of the estimates on $C(z, \zeta)$ (the estimate in Theorem 1.2 or the result by Christ, (4)) can be used in order to prove this theorem, because as soon as one supposes the compactness of the canonical solution operator $N$, the function $\rho$ turns out to be bounded and therefore (1) holds.

There is some natural gain (or loss) in the Hörmander estimates if the Laplacian of $\phi$ is big (or small). If we incorporate the Laplacian in the weight then we always get boundedness, under some mild regularity assumption (the doubling property) but we never get compactness:

Proposition 1.4. Let $\phi$ be a subharmonic function such that $\Delta \phi$ is doubling. The solution $u$ to the equation $\bar{\partial} u=f$ of minimal norm in $L^{2}\left(e^{-2 \phi}\right)$ is such that $\left\|u e^{-\phi}\right\|_{L^{p}(\mathbb{C})} \lesssim\left\|f e^{-\phi} \rho\right\|_{L^{p}(\mathbb{C})}$, for all $p \in[1, \infty]$. Moreover, the canonical solution operator $N$ acting from $L^{2}\left(e^{-2 \phi} \rho^{2}\right)$ to $L^{2}\left(e^{-2 \phi}\right)$ is always bounded but it is never compact.

Remark. The first statement in this proposition has been proved already in [MMO03, Theorem C] by using peak functions instead of estimates for the Bergman kernel.

\section{PRELIMinaires}

In this section we collect some material from [Chr91] and [MMO03] that will be used along the proofs and we deduce some easy estimates for the Bergman kernel near the diagonal.

Definition 2.1. A nonnegative Borel measure $\mu$ is called doubling if there exists $C>0$ such that

$$
\mu(D(z, 2 r)) \leq C \mu(D(z, r))
$$

for all $z \in \mathbb{C}$ and $r>0$. The smallest constant $C$ in the previous inequality is called the doubling constant for $\mu$. 
Lemma 2.2. [Chr91, Lemma 2.1] Let $\mu$ be a doubling measure in $\mathbb{C}$. There exists a constant $\gamma>0$ such that for any disks $D, D^{\prime}$ with respective radius $r>r^{\prime}$ and with $D \cap D^{\prime} \neq \emptyset$

$$
\left(\frac{\mu(D)}{\mu\left(D^{\prime}\right)}\right)^{\gamma} \lesssim \frac{r}{r^{\prime}} \lesssim\left(\frac{\mu(D)}{\mu\left(D^{\prime}\right)}\right)^{1 / \gamma}
$$

Remark. In particular for any $z \in \mathbb{C}$ and $r>1$ there exists a constant $\gamma>0$ (depending only on the doubling constant for $\mu$ ) such that

$$
r^{\gamma} \lesssim \mu\left(D^{r}(z)\right) \lesssim r^{1 / \gamma}
$$

It follows inmediately from Lemma 2.2 that the function $\rho$ is nearly constant on balls.

Lemma 2.3. If $D(z) \cap D(\zeta) \neq \emptyset$ then $\rho(z) \sim \rho(\zeta)$, with constants depending only on the doubling constant for $\Delta \phi$.

Remark. There exist constants $\eta, C>0$ and $0<\beta<1$ such that

$$
\frac{C^{-1}}{|z|^{\eta}} \leq \rho(z) \leq C|z|^{\beta}
$$

for $|z|>1$, [MMO03, Remark 1].

The following lemma shows that our main estimate (2) is symmetric in the variables $z, \zeta$.

Lemma 2.4. [Chr91, p. 205] If $\zeta \notin D(z)$ then

$$
\frac{\rho(z)}{\rho(\zeta)} \lesssim\left(\frac{|z-\zeta|}{\rho(\zeta)}\right)^{1-\delta}
$$

for some $0<\delta<1$ depending only on the doubling constant for $\Delta \phi$.

Definition 2.5. Given $z, \zeta \in \mathbb{C}$

$$
d_{\phi}(z, \zeta)=\inf _{\gamma} \int_{0}^{1}\left|\gamma^{\prime}(t)\right| \frac{d t}{\rho(\gamma(t))}
$$

where $\gamma$ runs on the piecewise $\mathcal{C}^{1}$ curves $\gamma:[0,1] \rightarrow \mathbb{C}$ with $\gamma(0)=z$ and $\gamma(1)=\zeta$.

The following lemma was proved in [MMO03, Lemma 4].

Lemma 2.6. There exists $\delta>0$ such that for every $r>0$ there exists $C_{r}>0$ such that

$$
C_{r}^{-1} \frac{|z-\zeta|}{\rho(z)} \leq d_{\phi}(z, \zeta) \leq C_{r} \frac{|z-\zeta|}{\rho(z)}, \quad \text { for } \zeta \in D^{r}(z)
$$

and

$$
C_{r}^{-1}\left(\frac{|z-\zeta|}{\rho(z)}\right)^{\delta} \leq d_{\phi}(z, \zeta) \leq C_{r}\left(\frac{|z-\zeta|}{\rho(z)}\right)^{2-\delta}, \quad \text { for } \zeta \in D^{r}(z)^{c} .
$$

The following lemma will be used repeatedly in what follows. 
Lemma 2.7. Let $\phi$ be a subharmonic function with $\mu=\Delta \phi$ doubling. Then for any $\epsilon>0$ and $k \geq 0$

$$
\int_{\mathbb{C}} \frac{|z-\zeta|^{k}}{\exp d_{\phi}(z, \zeta)^{\epsilon}} d \mu(z) \leq C \rho^{k}(\zeta),
$$

where $C>0$ is a constant depending only on $k, \epsilon$, and on the doubling constant for $\mu$.

Proof. Let $f(t)=t^{\frac{k}{\epsilon}}-\frac{k}{\epsilon} t^{\frac{k}{\epsilon}-1}$ then for any $x>0$

$$
\int_{x}^{+\infty} e^{-t} f(t) d t=e^{-x} x^{k / \epsilon}
$$

and

$$
\begin{aligned}
& \int_{\mathbb{C}} \frac{|z-\zeta|^{k}}{\exp d_{\phi}(z, \zeta)^{\epsilon}} d \mu(z) \lesssim \rho^{k}(\zeta) \mu(D(\zeta))+\int_{D(\zeta)^{c}} \rho^{k}(\zeta) \int_{\left(\frac{|z-\zeta|}{\rho(\zeta)}\right)^{\epsilon}}^{+\infty} e^{-t} f(t) d t d \mu(z) \\
& \quad \lesssim \rho^{k}(\zeta)+\rho^{k}(\zeta) \int_{1}^{+\infty} e^{-t} f(t) \mu\left(D^{t^{1 / \epsilon}}(\zeta)\right) d t \lesssim \rho^{k}(\zeta)\left(1+\int_{1}^{+\infty} e^{-t} f(t) t^{1 / \gamma \epsilon} d t\right)
\end{aligned}
$$

We will also use some Cauchy-type estimates for functions in the space,

Lemma 2.8. [MMO03, Lemma 19] For any $r>0$ there exists $C=C(r)>0$ such that for any $f \in \mathcal{H}(\mathbb{C})$ and $z \in \mathbb{C}:$
(a) $|f(z)|^{2} e^{-2 \phi(z)} \leq C \int_{D^{r}(z)}|f(\zeta)|^{2} e^{-2 \phi(\zeta)} \frac{d m(\zeta)}{\rho^{2}(\zeta)}$.
(b) $\left|\nabla\left(|f| e^{-\phi}\right)(z)\right|^{2} \leq \frac{C}{\rho^{2}(z)} \int_{D^{r}(z)}|f(\zeta)|^{2} e^{-2 \phi(\zeta)} \frac{d m(\zeta)}{\rho^{2}(\zeta)}$.
(c) If $s>r,|f(z)|^{2} e^{-2 \phi(z)} \leq C_{r, s} \int_{D^{s}(z) \backslash D^{r}(z)}|f(\zeta)|^{2} e^{-2 \phi(\zeta)} \frac{d m(\zeta)}{\rho^{2}(\zeta)}$.

The following result proved in [MMO03, Theorem 14] shows that the same space $\mathcal{F}_{\phi}^{2}$ can be defined with a more regular weight.

Proposition 2.9. Let $\phi$ be a subharmonic function such that $\Delta \phi$ is doubling. There exists $\widetilde{\phi} \in$ $\mathcal{C}^{\infty}(\mathbb{C})$ such that $|\phi-\widetilde{\phi}| \leq C$ with $\Delta \widetilde{\phi}$ doubling and

$$
\Delta \widetilde{\phi} \sim \frac{1}{\rho_{\widetilde{\phi}}^{2}} \sim \frac{1}{\rho_{\phi}^{2}} .
$$

As a first step in proving Theorem 1.1, in the remainder of the section we derive some estimates for the Bergman kernel on the diagonal or near the diagonal.

Proposition 2.10. There exist $C>0$ such that

$$
C^{-1} \frac{e^{2 \phi(z)}}{\rho^{2}(z)} \leq K(z, z) \leq C \frac{e^{2 \phi(z)}}{\rho^{2}(z)}
$$


Proof. Let $z \in \mathbb{C}$ be fixed. For any $M \in \mathbb{N}$ there exists a holomorphic function $P_{z}$ such that $P_{z}(z)=1$ and

$$
\left|P_{z}(\zeta)\right| \lesssim e^{\phi(\zeta)-\phi(z)} \min \left\{1,\left(\frac{\rho(z)}{|z-\zeta|}\right)^{M}\right\}
$$

see [MMO03, Appendix]. For some $c_{0}>0$ (to be determined) we define the entire function

$$
f_{z}(\zeta)=c_{0} \frac{e^{\phi(z)}}{\rho(z)} P_{z}(\zeta)
$$

Then

$$
\int_{\mathbb{C}}\left|f_{z}(\zeta)\right|^{2} e^{-2 \phi(\zeta)} d m(\zeta) \leq C c_{0}^{2}+\int_{D(z)^{c}}\left(\frac{\rho(z)}{|z-\zeta|}\right)^{2 M} \frac{d m(\zeta)}{\rho^{2}(z)}=C c_{0}^{2}\left(1+\frac{\pi}{M-1}\right) \leq 1
$$

for $c_{0}$ small enough. For such a fixed $c_{0}$ we have $f_{z}(z)=c_{0} e^{\phi(z)} \rho^{-1}(z)$ and therefore

$$
K(z, z)=\sup \left\{|f(z)|^{2}: f \in \mathcal{F}_{\phi}^{2},\|f\|_{\mathcal{F}_{\phi}^{2}} \leq 1\right\} \gtrsim \frac{e^{2 \phi(z)}}{\rho^{2}(z)} .
$$

The other estimate follows by using the reproducing property for the Bergman kernel, Lemma 2.3 and inequality (a) in Lemma 2.8, see the next proposition, where this is done in detail.

The following coarse estimate will give us (2) when the points $z, \zeta \in \mathbb{C}$ are close to each other.

Proposition 2.11. Let $K(z, \zeta)$ be the Bergman kernel for $\mathcal{F}_{\phi}^{2}$. Then there exists $C>0$ (depending only on the doubling constant for $\Delta \phi$ ) such that for any $z, \zeta \in \mathbb{C}$

$$
|K(z, \zeta)| \leq C \frac{e^{\phi(z)+\phi(\zeta)}}{\rho(z) \rho(\zeta)}
$$

Moreover there is an $r>0$ such that

$$
|K(z, \zeta)| \gtrsim \frac{e^{\phi(z)+\phi(\zeta)}}{\rho(z) \rho(\zeta)}, \quad \forall \zeta \in D^{r}(z) .
$$

Proof. Let $z \in \mathbb{C}$ be fixed. Applying (a) in Lemma 2.8 to the reproducing kernel $K_{z}$ and using Lemma 2.3

$$
\begin{aligned}
\left|K_{z}(\zeta)\right|^{2} e^{-2 \phi(\zeta)} & \lesssim \int_{D(\zeta)}\left|K_{z}(w)\right|^{2} e^{-2 \phi(w)} \frac{d m(w)}{\rho^{2}(w)} \lesssim \\
& \lesssim \int_{\mathbb{C}}\left|K_{z}(w)\right|^{2} e^{-2 \phi(w)} \frac{d m(w)}{\rho^{2}(\zeta)}=\frac{K(z, z)}{\rho^{2}(\zeta)} .
\end{aligned}
$$

By using Proposition 2.10 the estimate $(8)$ follows. Finally again by Proposition 2.10 the claim (9) holds when $z=\zeta$. Moreover Lemma 2.8(b) implies that for all $\zeta \in D^{r}(z)$,

$$
|| K_{z}(\zeta)\left|e^{-\phi(\zeta)}-\right| K_{z}(z)\left|e^{-\phi(z)}\right| \leq C e^{\phi(z)}|z-\zeta| / \rho^{3}(z) \leq C r e^{\phi(z)} / \rho^{2}(z),
$$

and if we pick $r$ small enough then (9) follows. 


\section{Proof of Theorem 1.1}

One technical difficulty is that our function $\phi$ is not smooth enough, so first of all we define a regularized version.

Let $0<\epsilon<1$ a constant to be chosen later. Let

$$
\varphi(w)=\left(\frac{|w-\zeta|}{\rho_{\phi}(\zeta)}\right)^{\epsilon}
$$

(we will write $\varphi_{\epsilon}$ if we need to stress the dependence on $\epsilon$ ). The function $\varphi$ is subharmonic and

$$
\frac{\partial \varphi}{\partial w}(w)=\frac{\epsilon|w-\zeta|^{\epsilon-2}(\bar{w}-\bar{\zeta})}{2 \rho_{\phi}^{\epsilon}(\zeta)}, \quad \Delta \varphi(w)=\frac{\epsilon^{2}|w-\zeta|^{\epsilon-2}}{4 \rho_{\phi}^{\epsilon}(\zeta)} .
$$

Considering the dependence on $\epsilon$ one has

$$
\Delta \varphi_{2 \epsilon}(w)=4\left|\frac{\partial \varphi_{\epsilon}}{\partial w}(w)\right|^{2}
$$

The Laplacian of $\varphi$ is not bounded above, so we define

$$
\psi=\frac{1}{\pi \rho_{\phi}^{2}(\zeta)} \chi_{\rho_{\phi}(\zeta)} * \varphi
$$

where $\chi_{\rho_{\phi}(\zeta)}=\chi_{D\left(0, \rho_{\phi}(\zeta)\right)}$ is the characteristic function of $D\left(0, \rho_{\phi}(\zeta)\right)$.

By Hölder's inequality

$$
\left|\frac{\partial \psi}{\partial w}\right|^{2} \leq \frac{1}{\pi \rho_{\phi}^{2}(\zeta)} \chi_{\rho_{\phi}(\zeta)} *\left|\frac{\partial \varphi}{\partial w}\right|^{2}
$$

and

$$
\Delta \psi_{2 \epsilon}(w)=\left(\frac{1}{\pi \rho_{\phi}^{2}(\zeta)} \chi_{\rho_{\phi}(\zeta)} * \Delta \varphi_{2 \epsilon}\right)(w)=\left(\frac{1}{\pi \rho_{\phi}^{2}(\zeta)} \chi_{\rho_{\phi}(\zeta)} * 4\left|\frac{\partial \varphi_{\epsilon}}{\partial w}\right|^{2}\right)(w)
$$

We denote $\Phi_{\epsilon}(w)=\Delta \psi_{2 \epsilon}(w) / 4$.

Let $\widetilde{\phi} \in \mathcal{C}^{\infty}(\mathbb{C})$ the regularized version of $\phi$ given by Proposition 2.9

Lemma 3.1. There exist $0<\epsilon_{0}<1$ and $0<C_{1}, C_{2}<1$ (depending only on the doubling constant for $\Delta \phi$ ) such that for any $0<\epsilon \leq \epsilon_{0}$

$$
\left|\frac{\partial \psi_{\epsilon}}{\partial w}(w)\right|^{2} \leq C_{1} \Delta \widetilde{\phi}(w), \quad \text { and } \quad \Delta \psi_{\epsilon}(w) \leq C_{2} \Delta \widetilde{\phi}(w) .
$$

This lemma is an easy consequence of the following:

Lemma 3.2. For any $C>0$ there exists $0<\epsilon_{0}<1$ (depending only on the doubling constant for $\Delta \phi$ and $C$ ) such that

$$
\Phi_{\epsilon}(w) \leq C \frac{1}{\rho_{\phi}^{2}(w)}
$$

if $0<\epsilon \leq \epsilon_{0}$. 
Lemma 3.2 implies Lemma 3.1. By (6) there exists $C^{\prime}>0$ such that

$$
\frac{1}{\rho_{\phi}^{2}(w)} \leq C^{\prime} \Delta \widetilde{\phi}(w) \text {. }
$$

Let $\epsilon_{0}>0$ the one provided by Lemma 3.2 for $C>0$ such that $4 C C^{\prime}<1$. If $0<\epsilon \leq \epsilon_{0}$ we have

and

$$
\left|\frac{\partial \psi_{\epsilon}}{\partial w}(w)\right|^{2} \leq \Phi_{\epsilon}(w) \leq C \frac{1}{\rho_{\phi}^{2}(w)} \leq C C^{\prime} \Delta \widetilde{\phi}(w),
$$

$$
\Delta \psi_{\epsilon}(w)=4 \Phi_{\epsilon / 2}(w) \leq 4 C \frac{1}{\rho_{\phi}^{2}(w)} \leq 4 C C^{\prime} \Delta \widetilde{\phi}(w) .
$$

Then it is enough to take $C_{1}=C C^{\prime}$ and $C_{2}=4 C C^{\prime}$.

Lemma 3.2 We want to see that for $C>0$ there exists $1>\epsilon_{0}>0$ such that for $0<\epsilon \leq \epsilon_{0}$

$$
\left(\frac{1}{\pi \rho_{\phi}^{2}(\zeta)} \chi_{\rho_{\phi}(\zeta)} *\left|\frac{\partial \varphi}{\partial w}\right|^{2}\right)(w) \leq C \frac{1}{\rho_{\phi}^{2}(w)} .
$$

We will split the proof in two cases:

CASE 1: Suppose that $D(w) \cap D^{2}(\zeta) \neq \emptyset$. The function $\Phi_{\epsilon}$ has a maximum in $w=\zeta$ (because $\left.|\partial \varphi / \partial w|^{2}(u) \sim 1 /|u-\zeta|^{2-2 \epsilon}\right)$ so it is enough to see that $\Phi_{\epsilon}(\zeta) \leq C \rho_{\phi}^{-2}(w)$. But

$$
\begin{aligned}
\Phi_{\epsilon}(\zeta) & =\frac{1}{\pi \rho^{2}(\zeta)} \int_{D(0, \rho(\zeta))} \frac{\epsilon^{2}|\zeta-z-\zeta|^{2 \epsilon-2}}{4 \rho^{2 \epsilon}(\zeta)} d m(z) \\
& =\frac{\epsilon^{2}}{4 \pi \rho^{2 \epsilon+2}(\zeta)} \int_{D(0, \rho(\zeta))}|z|^{2 \epsilon-2} d m(z)=\frac{\epsilon^{2}}{4 \pi \rho^{2 \epsilon+2}(\zeta)} \int_{0}^{\rho(\zeta)} \int_{0}^{2 \pi} t^{2 \epsilon-1} d t d \theta=\frac{\epsilon}{4 \rho^{2}(\zeta)},
\end{aligned}
$$

so we need

$$
\frac{\epsilon}{4} \lesssim\left(\frac{\rho(\zeta)}{\rho(w)}\right)^{2}
$$

and this property holds for $0<\epsilon \leq \epsilon_{0}$ because $\rho(\zeta) \sim \rho(w)$.

CASE 2: Suppose that $D(w) \cap D^{2}(\zeta)=\emptyset$. Then

$$
\begin{aligned}
\Phi_{\epsilon}(w) & =\frac{1}{\pi \rho^{2}(\zeta)} \int_{D(0, \rho(\zeta))} \frac{\epsilon^{2}|w-z-\zeta|^{2 \epsilon-2}}{4 \rho^{2 \epsilon}(\zeta)} d m(z) \\
& =\frac{\epsilon^{2}}{4 \pi \rho^{2+2 \epsilon}(\zeta)} \int_{D(0, \rho(\zeta))}|w-u|^{2 \epsilon-2} d m(u) \leq \frac{\epsilon^{2} 2^{2-2 \epsilon}}{4 \rho^{2 \epsilon}(\zeta)|w-\zeta|^{2-2 \epsilon}} .
\end{aligned}
$$

So we need

or equivalently

$$
\frac{\epsilon^{2}|w-\zeta|^{2 \epsilon-2}}{2^{2 \epsilon} \rho^{2 \epsilon}(\zeta)} \lesssim \frac{1}{\rho^{2}(w)}
$$

$$
\frac{2^{\epsilon} C}{\epsilon}\left(\frac{|w-\zeta|}{\rho(\zeta)}\right)^{1-\epsilon} \geq \frac{\rho(w)}{\rho(\zeta)}
$$


and this follows from Lemma 2.4 because $\zeta \notin D(w)$. We would like to mention that, as $|w-\zeta|>$ $\rho(\zeta)$, the last inequality holds also for any exponent smaller than the $\delta$ appearing in Lemma 2.4 . Finally, as $2^{\epsilon} C / \epsilon$ goes to infinity when $\epsilon \rightarrow 0$, one can find $\epsilon_{0}$ such that $(10)$ holds for any $0<\epsilon \leq \epsilon_{0}$.

From now on we will fix $\varepsilon>0$ in such a way that the conclusions of Lemma 3.1 do hold. The following lemma is an easy consequence of the previous ones.

Lemma 3.3. For $\varrho=\widetilde{\phi}-\psi$, one has

$$
\Delta \varrho \sim \Delta \widetilde{\phi}, \quad \text { and } \quad \frac{1}{\rho_{\varrho}^{2}} \sim \frac{1}{\rho_{\widetilde{\phi}}^{2}} .
$$

Proof. As $\psi$ is subharmonic $\Delta \widetilde{\phi} \geq \Delta \widetilde{\phi}-\Delta \psi=\Delta \varrho$. The other inequality follows from Lemma 3.1 since $\Delta \varrho \geq\left(1-C_{2}\right) \Delta \widetilde{\phi}$, with $0<C_{2}<1$. The relation between the corresponding regularization follows automatically.

The proof of Theorem 1.1. We will follow a similar argument as in [Lin01] when Lindholm studies the case when $\Delta \phi$ is bounded. In fact the basic trick goes back to Kerzman in [Ker72], where the Bergman kernel is estimated using the estimates on the solution to an inhomogeneous Cauchy-Riemann equation.

We are interested in studying the behaviour of $K(z, \zeta)$ when the points $z, \zeta$ are far apart.

Let $z, \zeta \in \mathbb{C}$ be fixed points such that $D(z) \cap D(\zeta)=\emptyset$. Let $0 \leq \chi \leq 1$ be a function in $\mathcal{C}_{c}^{\infty}(\mathbb{C})$ with supp $\chi \subset D(\zeta)$ such that $\chi \equiv 1$ in $D^{1 / 2}(\zeta)$ and

$$
|\bar{\partial} \chi|^{2} \lesssim \frac{\chi}{\rho^{2}(\zeta)}
$$

We have that

$$
\begin{aligned}
\left|K_{z}(\zeta)\right|^{2} e^{-2 \phi(\zeta)} & \lesssim \frac{1}{\rho^{2}(\zeta)} \int_{D^{1 / 2}(\zeta)}\left|K_{z}(w)\right|^{2} e^{-2 \phi(w)} d m(w) \\
= & \frac{1}{\rho^{2}(\zeta)} \int_{D^{1 / 2}(\zeta)} \chi(w)\left|K_{z}(w)\right|^{2} e^{-2 \phi(w)} d m(w) \lesssim \frac{1}{\rho^{2}(\zeta)}\left\|K_{z}\right\|_{L^{2}\left(\chi e^{-2 \phi}\right)}^{2}
\end{aligned}
$$

Then $\left\|K_{z}\right\|_{L^{2}\left(\chi e^{-2 \phi}\right)}=\sup _{f}\left|\left\langle f, K_{z}\right\rangle_{L^{2}\left(\chi e^{-2 \phi}\right)}\right|$ where the supremum runs over all the holomorphic functions $f$ in $D(\zeta)$ such that

$$
\int|f|^{2} e^{-2 \phi} \chi d m=1
$$

As $f \chi \in L^{2}\left(e^{-2 \phi}\right)$ one has

$$
\left\langle f, K_{z}\right\rangle_{L^{2}\left(\chi e^{-2 \phi}\right)}=P(f \chi)(z),
$$

where $P=P_{\phi}$ stands for the Bergman projection

$$
P_{\phi}(f)(z)=\int_{\mathbb{C}} K(z, \zeta) f(\zeta) e^{-2 \phi(\zeta)} d m(\zeta),
$$


which is bounded from $L^{2}\left(e^{-2 \phi}\right)$ to $\mathcal{F}_{\phi}^{2}$. Now

$$
u=f \chi-P(f \chi),
$$

is the canonical solution (in $L^{2}\left(e^{-2 \phi}\right)$ ) of

$$
\bar{\partial} u=\bar{\partial}(f \chi)=f \bar{\partial} \chi
$$

and, since $\chi(z)=0$, one has

$$
\left|\left\langle f, K_{z}\right\rangle_{L^{2}\left(\chi e^{-2 \phi}\right)}\right|=|P(f \chi)(z)|=|u(z)| .
$$

As $D_{\phi}(\zeta) \cap D_{\phi}(z)=\emptyset$, the function $f \chi$ vanishes off $D_{\phi}(\zeta)$ and therefore (recall that $\rho_{\varrho} \sim \rho_{\widetilde{\phi}} \sim$ $\rho_{\phi}$ by Lemma 3.3 the function $u$ is holomorphic in $D_{\varrho}^{r}(z)$ for some $r>0$, so by Lemma 2.8 (a)

$$
\begin{aligned}
|u(z)|^{2} e^{-2 \phi(z)+2 \psi(z)} & \lesssim|u(z)|^{2} e^{-2 \widetilde{\phi}(z)+2 \psi(z)}=|u(z)|^{2} e^{-2 \varrho(z)} \\
& \lesssim \int_{D_{\varrho}^{r}(z)}|u(w)|^{2} e^{-2 \varrho(w)} \frac{d m(w)}{\rho_{\varrho}^{2}(w)} \lesssim \frac{1}{\rho_{\varrho}^{2}(z)} \int_{\mathbb{C}}|u(w)|^{2} e^{-2 \varrho(w)} d m(w) \\
& \sim \frac{1}{\rho_{\phi}^{2}(z)} \int_{\mathbb{C}}|u(w)|^{2} e^{-2 \varrho(w)} d m(w) .
\end{aligned}
$$

We estimate this last integral using the classical Hörmander theorem:

Theorem 3.4 (Hörmander). Let $\Omega \subset \mathbb{C}$ be a domain and $\phi \in \mathcal{C}^{2}(\Omega)$ be such that $\Delta \phi \geq 0$. For any $f \in L_{\text {loc }}^{2}(\Omega)$ there exist a solution $u$ to $\bar{\partial} u=f$ such that

$$
\int|u|^{2} e^{-2 \phi} \leq \int \frac{|f|^{2}}{\Delta \phi} e^{-2 \phi}
$$

and also a variant due to Berndtsson:

Theorem 3.5. [Ber01, Lemma 2.2] If

$$
\left|\frac{\partial \psi}{\partial w}\right|^{2} \leq C_{1} \Delta \widetilde{\phi}, \quad \text { with } \quad 0<C_{1}<1
$$

and for any $g$ one can find $v$ such that $\bar{\partial} v=g$ with

$$
\int|v|^{2} e^{-2 \phi-2 \psi} \leq \int \frac{|g|^{2}}{\Delta \widetilde{\phi}} e^{-2 \phi-2 \psi},
$$

then for the canonical solution $v_{0}$ in $L^{2}\left(e^{-2 \phi}\right)$, one has

$$
\int\left|v_{0}\right|^{2} e^{-2 \phi+2 \psi} \leq C \int \frac{|g|^{2}}{\Delta \widetilde{\phi}} e^{-2 \phi+2 \psi},
$$

where $C=6 /\left(1-C_{1}\right)^{2}$. 
We know that $\Delta(\widetilde{\phi}+\psi) \geq 0$, then applying Theorem 3.4 to $\bar{\partial}(f \chi)$, one has $v$ such that $\bar{\partial} v=\bar{\partial}(f \chi)$ with

$$
\begin{aligned}
\int|v|^{2} e^{-2 \widetilde{\phi}-2 \psi} & \leq \int \frac{|\bar{\partial} v|^{2}}{\Delta(\widetilde{\phi}+\psi)} e^{-2 \widetilde{\phi}-2 \psi} \\
& \leq \int \frac{|\bar{\partial} v|^{2}}{\Delta \widetilde{\phi}} e^{-2 \widetilde{\phi}-2 \psi} .
\end{aligned}
$$

As $|\phi-\widetilde{\phi}| \leq C$ we have that $(13)$ holds and by Theorem 3.5

$$
\int|u|^{2} e^{-2 \phi+2 \psi} \leq C \int \frac{|\bar{\partial} u|^{2}}{\Delta \widetilde{\phi}} e^{-2 \phi+2 \psi} .
$$

The functions $\phi, \widetilde{\phi}$ are pointwise equivalent and $\Delta \widetilde{\phi} \sim \rho_{\phi}^{-2}$ so one can estimate (12) as

$$
\begin{aligned}
\frac{1}{\rho_{\phi}^{2}(z)} \int|u(w)|^{2} & e^{-2 \varrho(w)} d m(w) \lesssim \frac{1}{\rho_{\phi}^{2}(z)} \int_{D(\zeta)} \rho_{\phi}^{2}(w)|\bar{\partial}(f \chi)(w)|^{2} e^{-2 \varrho(w)} d m(w) \\
& \lesssim \frac{1}{\rho_{\phi}^{2}(z)} \int_{D(\zeta)} \rho_{\phi}^{2}(w)|f(w)|^{2} \frac{\chi(w)}{\rho_{\phi}^{2}(\zeta)} e^{-2 \varrho(w)} d m(w) .
\end{aligned}
$$

The function $\psi$ is bounded above in $D(\zeta)$ by a constant depending only on the doubling constant for $\Delta \phi$, indeed, for $w \in D(\zeta)$

$$
\frac{1}{\pi \rho_{\phi}^{2}(\zeta)} \int \chi_{\rho_{\phi}(\zeta)}(w-u) \varphi(u) d m(u) \leq \frac{1}{\pi \rho_{\phi}^{2}(\zeta)} \int_{D^{2}(\zeta)} \varphi(u) d m(u) \lesssim 2^{\epsilon} .
$$

So finally (14) can be estimated by

$$
\int_{D(\zeta)} \frac{\rho_{\phi}^{2}(w)|f(w)|^{2} \chi(w)}{\rho_{\phi}^{2}(z) \rho_{\phi}^{2}(\zeta)} e^{-2 \varrho(w)} d m(w) \lesssim \int_{D(\zeta)} \frac{|f(w)|^{2} \chi(w)}{\rho_{\phi}^{2}(z)} e^{-2 \phi(w)} d m(w)=\frac{1}{\rho_{\phi}^{2}(z)} .
$$

and we have

$$
|K(\zeta, z)|^{2} \lesssim \frac{1}{\rho_{\phi}^{2}(z) \rho_{\phi}^{2}(\zeta)} \frac{e^{2 \phi(z)+2 \phi(\zeta)}}{e^{2 \psi(z)}}
$$

3.1. Pointwise estimates. In this subsection we deduce a new expression, without $\psi$, for (15). The new expression is the one appearing in Theorem 1.1 and therefore this will finish the proof.

Lemma 3.6. If $D(\zeta) \cap D(w)=\emptyset$ there exists $C>0$ such that

$$
|\psi(w)-\varphi(w)| \leq C
$$

Proof. Using the subharmonicity

$$
\psi(w)-\varphi(w)=\frac{1}{\rho^{\epsilon}(\zeta)}\left\{\frac{1}{\pi \rho_{\phi}^{2}(\zeta)} \int_{D(\zeta)}|w-u|^{\epsilon} d m(u)-|w-\zeta|^{\epsilon}\right\} \geq 0 .
$$


On the other hand, if $|w-\zeta| \leq 2 \rho(\zeta)$ it is plain that

$$
\psi(w)=\frac{1}{\pi \rho^{2+\epsilon}(\zeta)} \int_{D(\zeta)}|w-u|^{\epsilon} d m(u) \leq 3^{\epsilon}
$$

and therefore $0 \leq \psi(w)-\varphi(w) \leq 3^{\epsilon}$.

For $|w-\zeta| \geq 2 \rho(\zeta)$ (we will write $v(z)=|w-z|^{\epsilon}$ ) we have

$$
\begin{aligned}
\psi(w)-\varphi(w) & =\frac{1}{\rho^{\epsilon}(\zeta)}\left\{\frac{1}{\pi \rho_{\phi}^{2}(\zeta)} \int_{D(\zeta)} v(u) d m(u)-v(\zeta)\right\} \\
& =\frac{1}{2 \pi \rho^{\epsilon}(\zeta)} \int_{D(\zeta)}\left\{\log \left(\frac{\rho(\zeta)}{|u-\zeta|}\right)+\frac{1}{2}\left(\left(\frac{|u-\zeta|}{\rho(\zeta)}\right)^{2}-1\right)\right\} \Delta v(u) d m(u) \\
& \leq \frac{1}{2 \pi \rho^{\epsilon}(\zeta)} \int_{D(\zeta)} \log \left(\frac{\rho(\zeta)}{|u-\zeta|}\right) \Delta v(u) d m(u),
\end{aligned}
$$

for the second equality see [BO97, section 3.3.]. By [MMO03, Lemma 5] the last integral is smaller than

$$
\frac{1}{2 \pi \rho^{\epsilon}(\zeta)} \int_{D(\zeta)} \Delta v(u) d m(u)
$$

times a constant depending only on the doubling constant for $\Delta v$ (which in turn depends only on $\epsilon)$. For any $u \in D(\zeta)$ one deduces from $|w-\zeta| \geq 2 \rho(\zeta)$ that $|u-w| \geq \rho(\zeta)$, and

$$
\int_{D(\zeta)} \Delta v(u) d m(u) \leq\left(\frac{\epsilon}{2}\right)^{2} \frac{1}{\rho^{2-\epsilon}}(\zeta) m(D(\zeta))
$$

so finally

$$
\psi(w)-\varphi(w) \lesssim \frac{\epsilon^{2}}{8}
$$

\section{Proof of Theorems 1.2 And 1.3}

Theorem 1.2 Let $r>0$ be as in Proposition 2.11. Let $\left\{z_{i}\right\}$ be a sequence of points in $\mathbb{C}$ such that $\left\{D^{r}\left(z_{j}\right)\right\}$ is a covering of $\mathbb{C}$. Let $\left\{\chi_{i}\right\}$ be a partition of unity subordinate to the covering. Let $k_{z}(\zeta)=\overline{K(z, \zeta)} / \sqrt{K(z, z)}$ be the normalized reproducing kernel in $\mathcal{F}_{\phi}^{2}$. Consider the operator

$$
L^{2}\left(e^{-2 \phi}\right) \ni f \mapsto u_{i}(z)=k_{z_{i}}(z) \int_{\mathbb{C}} \frac{f(\zeta) \chi_{i}(\zeta)}{(\zeta-z) k_{z_{i}}(\zeta)} d m(\zeta)
$$

By Cauchy-Pompeiu formula one has that $\bar{\partial} u_{i}=f \chi_{i}$. Then the kernel

$$
G(z, \zeta)=\left(\sum_{i} \frac{k_{z_{i}}(z) \chi_{i}(\zeta)}{(\zeta-z) k_{z_{i}}(\zeta)}\right) e^{\phi(\zeta)-\phi(z)}
$$

is such that

$$
u(z)=\int_{\mathbb{C}} e^{\phi(z)-\phi(\zeta)} G(z, \zeta) f(\zeta) d m(\zeta)
$$


solves $\bar{\partial} u=f$. Let $z \in \mathbb{C}$ be fixed and $|z-\zeta| \leq R \rho(z)$ for some fixed $R \gg 0$, then there is a finite number of balls of the covering intersecting $D^{R}(z)$ and by Proposition 2.11 one has

$$
|G(z, \zeta)| \lesssim|z-\zeta|^{-1}
$$

Also when $|z-\zeta| \geq R \rho(z)$ there is a finite number of balls in the covering containing $\zeta$ and this will give us a finite number of summands in $G$. For one of these terms summands one has by Theorem 1.1 that

$$
\left|k_{z_{i}}(z)\right| \lesssim \frac{e^{\phi(z)}}{\rho(z) \exp d_{\phi}\left(z, z_{i}\right)^{\epsilon}}
$$

and

$$
\left|\frac{k_{z_{i}}(z) \chi_{i}(\zeta)}{(\zeta-z) k_{z_{i}}(\zeta)}\right| e^{\phi(\zeta)} \lesssim \frac{e^{\phi(\zeta)-\phi(z)} e^{\phi(z)}}{\exp d_{\phi}\left(z, z_{i}\right)^{\epsilon} \rho^{2}(z) \rho^{-1}(\zeta) e^{\phi(\zeta)}} \lesssim \frac{1}{\rho(z) \exp d_{\phi}\left(z, z_{i}\right)^{\epsilon}},
$$

but as $d_{\phi}\left(z, z_{i}\right) \sim d_{\phi}(z, \zeta)$ this gives us the estimate of $G$.

Now we want to show that the same estimate holds for the kernel $C$. If $N$ is the canonical solution operator and $M$ is the solution operator given by the kernel $G$ above, one can see that $N=M-P M$ where $P$ stands for the Bergman projection. Then for $f \in \mathcal{F}_{\phi}^{2}$

$$
N f(z)=\int_{\mathbb{C}} C(z, \zeta) e^{\phi(z)-\phi(\zeta)} f(\zeta) d m(\zeta)
$$

where

$$
C(z, \zeta)=G(z, \zeta)-e^{-\phi(z)} \int_{\mathbb{C}} K(z, \xi) G(\xi, \zeta) e^{-\phi(\xi)} d m(\xi)
$$

Suppose first that $|z-\zeta| \leq \rho(z)$. We split the last integral and use the estimates on $G$ and the Bergman kernel

$$
\begin{aligned}
& \int_{\mathbb{C}}|K(z, \xi) G(\xi, \zeta)| e^{-(\phi(\xi)+\phi(z))} d m(\xi) \lesssim \frac{1}{\rho(z)} \int_{D^{K}(\zeta)} \rho^{-1}(\zeta)|\xi-\zeta|^{-1} d m(\xi) \\
& \quad+\frac{1}{\rho^{2}(\zeta)} \int_{D^{K}(\zeta)^{c}} \frac{\rho^{-1}(\xi)}{\exp d_{\phi}(\xi, \zeta)^{\epsilon}} d m(\xi)
\end{aligned}
$$

and we get that the first integral is bounded by a constant, where $K>1$ is such that $D(z) \subset$ $D^{K}(\zeta)$. Now by Proposition 2.4 there exists $\epsilon^{\prime}>0$ such that

$$
\begin{aligned}
\int_{D^{K}(\zeta)^{c}} & \frac{\rho^{-1}(\xi)}{\exp d_{\phi}(\xi, \zeta)^{\epsilon}} d m(\xi) \lesssim \int_{D^{K}(\zeta)^{c} \cap\{\xi:|\zeta-\xi|<\rho(\xi)\}} \frac{\rho^{-1}(\zeta)}{\exp d_{\phi}(\xi, \zeta)^{\epsilon}} d m(\xi) \\
+ & \frac{\rho(\zeta)}{\operatorname{loxp} d_{\phi}(\xi, \zeta)^{\epsilon^{\prime}}} \frac{d m(\xi)}{\rho^{2}(\xi)} \lesssim \rho(\zeta),
\end{aligned}
$$

where for the first integral we use that $\{\xi:|\zeta-\xi|<\rho(\xi)\} \subset D^{K^{\prime}}(\zeta)$ for some $K^{\prime}>0$ and for the second one we use Lemma 2.7 together with Proposition 2.9 getting

$$
|C(z, \zeta)| \lesssim|z-\zeta|^{-1}, \text { when }|z-\zeta| \leq \rho(z) .
$$

For $|z-\zeta|>\rho(z)$ and given $0<\eta<1$ we split the integral in the regions defined by

(i) $d_{\phi}(\xi, \zeta) \leq \eta d_{\phi}(z, \zeta)$, 
(ii) $d_{\phi}(\xi, z) \leq \eta d_{\phi}(z, \zeta)$,

(iii) $d_{\phi}(\xi, \zeta)>\eta d_{\phi}(z, \zeta)$ and $d_{\phi}(\xi, z)>\eta d_{\phi}(z, \zeta)$.

In case $(i)$ we have $d_{\phi}(z, \zeta) \leq d_{\phi}(z, \xi)+d_{\phi}(\xi, \zeta) \leq d_{\phi}(z, \xi)+\eta d_{\phi}(z, \zeta)$ and $d_{\phi}(z, \xi) \leq d_{\phi}(z, \zeta)+$ $d_{\phi}(\xi, \zeta) \leq(1+\eta) d_{\phi}(z, \zeta)$ then

$$
(1-\eta) d_{\phi}(z, \zeta) \leq d_{\phi}(z, \xi) \leq(1+\eta) d_{\phi}(z, \zeta)
$$

and (recall that $|G(\xi, \zeta)| \lesssim \rho^{-1}(\zeta) \exp \left(-d_{\phi}(\xi, \zeta)^{\epsilon}\right)$ for $|\xi-\zeta| \geq \rho(\zeta)$ )

$$
\begin{aligned}
\int_{d_{\phi}(\xi, \zeta) \leq \eta d_{\phi}(z, \zeta)} \mid & K(z, \xi) G(\xi, \zeta) \mid e^{-(\phi(\xi)+\phi(z))} d m(\xi) \lesssim \frac{1}{\rho(z)} \int_{D^{\tau}(\zeta)} \frac{1}{\rho(\xi)|\xi-\zeta| \exp d_{\phi}(z, \xi)^{\epsilon}} d m(\xi) \\
& +\int_{\left\{d_{\phi}(\xi, \zeta) \leq \eta d_{\phi}(z, \zeta)\right\} \cap D^{\tau}(\zeta)^{c}} \frac{1}{\rho(\zeta) \rho(z) \rho(\xi) \exp \left(d_{\phi}(z, \xi)^{\epsilon}+d_{\phi}(z, \xi)^{\epsilon}\right)} d m(\xi) \\
& \lesssim \rho^{-1}(z) \exp \left(-d_{\phi}(z, \zeta)^{\epsilon}\right)\left(1+\frac{1}{\rho(\zeta)} \int_{D^{\tau}(\zeta)^{c}} \frac{\rho^{-1}(\xi)}{\exp d_{\phi}(z, \xi)^{\epsilon}} d m(\xi)\right),
\end{aligned}
$$

and the last integral can be bounded as above. An entirely analogous argument proves case (ii). Let $A$ be denote the region defined by (iii) (in the estimates which follow the value of the exponent $\epsilon$ may change from line to line although it is always strictly positive)

$$
\begin{aligned}
\int_{A}|K(z, \xi) G(\xi, \zeta)| e^{-(\phi(\xi)+\phi(z))} d m(\xi) \lesssim \frac{1}{\rho(\zeta) \rho(z)} \int_{A} \frac{\rho^{-1}(\xi)}{\exp \left(d_{\phi}(z, \xi)^{\epsilon}+d_{\phi}(\xi, \zeta)^{\epsilon}\right)} d m(\xi) \\
\quad \lesssim \frac{1}{\rho(\zeta) \rho(z)}\left(\int_{A \cap\left\{d_{\phi}(\xi, z) \leq d_{\phi}(\xi, \zeta)\right\}} \frac{\rho^{-1}(\xi)}{\exp 2 d_{\phi}(z, \xi) \epsilon} d m(\xi)+\int_{A \cap\left\{d_{\phi}(\xi, z) \geq d_{\phi}(\xi, \zeta)\right\}} \frac{\rho^{-1}(\xi)}{\exp 2 d_{\phi}(\xi, \zeta)^{\epsilon}} d m(\xi)\right) \\
\quad \lesssim \frac{1}{\rho(z) \rho(\zeta)} \int_{A} \frac{\rho(\xi)}{\exp d_{\phi}(z, \xi)^{\epsilon}} d \mu(\xi)+\frac{1}{\rho(z) \rho(\zeta)} \int_{A} \frac{\rho(\xi)}{\exp d_{\phi}(\xi, \zeta)^{\epsilon}} d \mu(\xi),
\end{aligned}
$$

now we have

$$
\begin{gathered}
\frac{1}{\rho(z)} \int_{A} \frac{\rho(\xi)}{\exp d_{\phi}(z, \xi)^{\epsilon}} d \mu(\xi) \lesssim \int_{A} \frac{1}{\exp d_{\phi}(z, \xi)^{\epsilon}} d \mu(\xi) \lesssim \int_{d_{\phi}(z, \xi)>\eta d_{\phi}(z, \zeta)} \int_{d_{\phi}(z, \xi)^{\epsilon}}^{+\infty} e^{-t} d t d \mu(\xi) \\
\lesssim \int_{\eta^{\epsilon} d_{\phi}(z, \zeta)^{\epsilon}}^{+\infty} \mu\left(\left\{\xi: d_{\phi}(z, \xi)<t^{1 / \epsilon}\right\}\right) e^{-t} d t \lesssim \int_{\eta^{\epsilon} d_{\phi}(z, \zeta)^{\epsilon}}^{+\infty} t^{\gamma} e^{-t} d t \lesssim \frac{1}{\exp d_{\phi}(z, \zeta)^{\epsilon}}
\end{gathered}
$$

where as before $\mu=\Delta \phi$.

Theorem 1.3. Let $\left\{z_{j}\right\}$ be a sequence of complex numbers such that $z_{j} \rightarrow \infty$ for $j \rightarrow \infty$. We want to show that $\rho\left(z_{j}\right) \rightarrow 0$ when $N$ is compact. Defining holomorphic $(0,1)$-forms $f_{j}$ and functions $u_{j}$ as

$$
f_{j}(z)=k_{z_{j}}(z) d \bar{z}, \quad u_{j}(z)=\overline{\left(z-z_{j}\right)} k_{z_{j}}(z),
$$

then $\bar{\partial} u_{j}=f_{j}$. Observe that $u_{j} \in L^{2}\left(e^{-2 \phi}\right)$ because of the above estimate, Lemma 2.7 and Proposition 2.9

$$
\int_{\mathbb{C}}\left|z-z_{j}\right|^{2}\left|k_{z_{j}}(z)\right|^{2} e^{-2 \phi(z)} d m(z) \lesssim \rho^{2}\left(z_{j}\right)<\infty
$$


Finally, as the reproducing kernels $\left\{k_{w}\right\}_{w \in \mathbb{C}}$ are dense in $\mathcal{F}_{\phi}^{2}$ and

$$
\left\langle u_{j}, k_{w}\right\rangle=\left\langle\left(z-z_{j}\right) k_{z_{j}}(z), k_{w}(z)\right\rangle=0,
$$

the solution $u_{j}$ is the canonical solution to $\bar{\partial}$ i.e. $u_{j}=N f_{j}$. By hypothesis, the operator $N$ is compact and $\left\|f_{j}\right\|=1$ therefore there exist a convergent subsequence of $\left\{u_{j}\right\}$ (which we denoted as before).

The functions $u_{j}$ are basically concentrated on $D\left(z_{j}\right)$. Indeed, by Proposition 2.11 one has $\left|k_{z_{j}}(z)\right| \lesssim \rho^{-1}\left(z_{j}\right) e^{\phi(z)}$ so

$$
\int_{D^{r}\left(z_{j}\right)}\left|\left(z-z_{j}\right) k_{z_{j}}(z)\right|^{2} e^{-2 \phi(z)} d m(z) \lesssim \frac{1}{\rho^{2}\left(z_{j}\right)} \int_{D^{r}\left(z_{j}\right)}\left|z-z_{j}\right|^{2} d m(z) \lesssim \rho^{2}\left(z_{j}\right)
$$

and conversely by Lemma 2.8 (c)

$$
\begin{aligned}
\int_{D^{r}\left(z_{j}\right)}\left|\left(z-z_{j}\right) k_{z_{j}}(z)\right|^{2} e^{-2 \phi(z)} d m(z) \gtrsim \int_{D^{r}\left(z_{j}\right) \backslash D^{r / 2}\left(z_{j}\right)}\left|\left(z-z_{j}\right) k_{z_{j}}(z)\right|^{2} e^{-2 \phi(z)} d m(z) \\
\quad \gtrsim \rho^{4}\left(z_{j}\right) \int_{D^{r}\left(z_{j}\right) \backslash D^{r / 2}\left(z_{j}\right)}\left|k_{z_{j}}(z)\right|^{2} e^{-2 \phi(z)} \frac{d m(z)}{\rho^{2}(z)} \gtrsim \rho^{4}\left(z_{j}\right)\left|k_{z_{j}}\left(z_{j}\right)\right|^{2} e^{-2 \phi\left(z_{j}\right)} \sim \rho^{2}\left(z_{j}\right) .
\end{aligned}
$$

In particular, just because the operator $N$ is bounded, the sequence $\left\{\rho\left(z_{j}\right)\right\}$ has to be bounded. Also by Lemma 2.7 and Proposition 2.9 one has

$$
\int_{D^{r}\left(z_{j}\right)^{c}}\left|\left(z-z_{j}\right) k_{z_{j}}(z)\right|^{2} e^{-2 \phi(z)} d m(z) \lesssim C_{r} \rho^{2}\left(z_{j}\right)
$$

where $C_{r} \rightarrow 0$ when $r \rightarrow \infty$.

The sequence $\left\{u_{j}\right\}$ is a Cauchy sequence so

$$
\left\|u_{j}-u_{k}\right\|^{2}=\left\|u_{j}\right\|^{2}+\left\|u_{k}\right\|^{2}+2 \operatorname{Re}\left\langle u_{j}, u_{k}\right\rangle \rightarrow 0,
$$

for $j, k \rightarrow \infty$. To complete this part of the proof we have to see that the scalar product is small also when $z_{j}$ and $z_{k}$ are far enough from each other. Indeed, given $\epsilon>0$ there exists $r_{\epsilon}$ such that for $r \geq r_{\epsilon}$

$$
\int_{D^{r}\left(z_{k}\right)^{c}}\left|\left(z-z_{k}\right) k_{z_{k}}(z)\right|^{2} e^{-2 \phi(z)} d z, \int_{D^{r}\left(z_{j}\right)^{c}}\left|\left(z-z_{j}\right) k_{z_{j}}(z)\right|^{2} e^{-2 \phi(z)} d z<\epsilon .
$$

Now let $\left|z_{j}-z_{k}\right| \gg r_{\epsilon} \max \left\{\rho\left(z_{j}\right), \rho\left(z_{k}\right)\right\}$. The $L^{2}-$ norm of $u_{j}$ on $D^{r}\left(z_{j}\right)$ is pointwise equivalent to $\rho\left(z_{j}\right)$ (and this value is bounded above) so applying Hölder's inequality to

$$
\begin{aligned}
& \left|\left\langle u_{j}, u_{k}\right\rangle\right| \leq \int_{\mathbb{C}}\left|z-z_{j}\right|\left|z-z_{k}\right|\left|k_{z_{j}}(z)\right|\left|k_{z_{k}}(z)\right| e^{-2 \phi(z)} d m(z) \\
& \lesssim\left[\int_{D^{r_{\epsilon}\left(z_{j}\right)}}+\int_{D^{r_{\epsilon}\left(z_{k}\right)}}+\int_{\mathbb{C} \backslash D^{r_{\epsilon}\left(z_{j}\right) \cup D^{r_{\epsilon}}\left(z_{k}\right)}}\right]\left|z-z_{j}\left\|z-z_{k}|| k_{z_{j}}(z)\right\| k_{z_{k}}(z)\right| e^{-2 \phi(z)} d m(z),
\end{aligned}
$$

we deduce that the scalar product is arbitrarily small and

$$
\rho^{2}\left(z_{j}\right) \sim\left\|u_{j}\right\|^{2} \rightarrow 0, \quad j \rightarrow \infty
$$


Suppose now that $\rho(z) \rightarrow 0$ when $|z| \rightarrow \infty$ and let

$$
M: L^{2}\left(e^{-2 \phi}\right) \rightarrow L^{2}\left(e^{-2 \phi}\right)
$$

be such a solution operator, i.e. $\bar{\partial} M f=f$. If $M$ is compact then the canonical solution operator will be compact because it can be written as $N=M-P M$ where $P$ is the Bergman projection.

So all we have to show is that there exists a solution operator for the $\bar{\partial}$ problem which is compact. First of all, the operator $M_{\delta}: L^{2}\left(e^{-2 \phi}\right) \rightarrow L^{2}\left(e^{-2 \phi}\right)$ defined as

$$
M_{\delta} f(z)=\int_{\left\{\zeta \in \mathbb{C}: d_{\phi}(z, \zeta)<\delta\right\}} G(z, \zeta) f(\zeta) e^{\phi(z)-\phi(\zeta)} d m(\zeta)
$$

has norm $O(\delta)$ as $\delta \rightarrow 0$. Indeed, let $z \in \mathbb{C}$ be fixed, then

$$
\left|M_{\delta} f(z) e^{-\phi(z)}\right| \leq\left\|f e^{-\phi}\right\|_{L^{\infty}(\mathbb{C})} \int_{|z-\zeta|<C \delta \rho(z)} \frac{1}{|z-\zeta|} d m(\zeta) \leq C \delta \rho(z)\left\|f e^{-\phi}\right\|_{L^{\infty}(\mathbb{C})}
$$

where the constant $C$ only depends on the doubling constant for $\Delta \phi$. Also

$$
\int_{\mathbb{C}}\left|M_{\delta} f(z)\right| e^{-\phi(z)} d m(z) \lesssim \delta\|\rho\|_{L^{\infty}(\mathbb{C})}\left\|f e^{-\phi}\right\|_{L^{1}(\mathbb{C})},
$$

and by the Marcinkiewicz interpolation theorem, when $\rho$ is bounded, the norm of the operator from $L^{2}\left(e^{-2 \phi}\right)$ to $L^{2}\left(e^{-2 \phi}\right)$ is $O(\delta)$.

We define now (for big $R>0$ ) the operator $M_{\delta}^{R}$ as

$$
M_{\delta}^{R} f(z)=\chi_{D(0, R)}(z) \int_{\left\{\zeta \in \mathbb{C}: \delta<d_{\phi}(z, \zeta)\right\}} G(z, \zeta) f(\zeta) e^{\phi(z)-\phi(\zeta)} d m(\zeta) .
$$

This operator is compact because it is Hilbert-Schmidt

$$
\begin{aligned}
\int_{D(0, R)} & \int_{\left\{\zeta \in \mathbb{C}: \delta<d_{\phi}(z, \zeta)\right\}}|G(z, \zeta)|^{2} d m(\zeta) d m(z) \\
& \lesssim \int_{D(0, R)} \frac{1}{\rho^{2}(z)} \int_{D^{\delta}(z)^{c}} \frac{1}{\exp \left(2 d_{\phi}(z, \zeta)^{\epsilon}\right)} d m(\zeta) d m(z) \leq O\left(R^{2}\right) .
\end{aligned}
$$

Finally, for big $R>0$, we define the operator $M^{R}$ as

$$
M^{R} f(z)=\chi_{D(0, R)^{c}}(z) \int_{\left\{\zeta \in \mathbb{C}: \delta<d_{\phi}(z, \zeta)\right\}} G(z, \zeta) f(\zeta) e^{\phi(z)-\phi(\zeta)} d m(\zeta)
$$

We can control its norm, because

$$
\left|M^{R} f(z) e^{-\phi(z)}\right| \lesssim \chi_{D(0, R)^{c}}(z) \rho(z)\left\|f e^{-\phi}\right\|_{L^{\infty}(\mathbb{C})}
$$

and therefore

$$
\left\|e^{-\phi} M^{R} f\right\|_{L^{\infty}(\mathbb{C})} \lesssim \sup _{|z| \geq R} \rho(z)\left\|f e^{-\phi}\right\|_{L^{\infty}(\mathbb{C})}
$$


For the $L^{1}$ norm

$$
\begin{gathered}
\int_{\mathbb{C}}\left|M^{R} f(z)\right| e^{-\phi(z)} d m(z) \lesssim \int_{D(0, R)^{c}} \frac{1}{\rho(z)} \int_{\mathbb{C}} \frac{1}{\exp \left(d_{\phi}(z, \zeta)^{\epsilon}\right)}|f(\zeta)| e^{-\phi(\zeta)} d m(\zeta) d m(z) \\
\lesssim\left(\sup _{|z| \geq R} \rho(z)\right) \int_{\mathbb{C}}|f(\zeta)| e^{-\phi(\zeta)} \int_{d_{\phi}(\zeta, z)>\delta} \frac{1}{\rho^{2}(z) \exp \left(d_{\phi}(z, \zeta)^{\epsilon}\right)} d m(z) d m(\zeta),
\end{gathered}
$$

the inner integral is finite again because of Lemma 2.7 combined with Proposition 2.9. Finally, by the Marcinkiewicz interpolation theorem

$$
\left\|e^{-\phi} M^{R} f\right\|_{L^{2}(\mathbb{C})} \lesssim \sup _{|z| \geq R} \rho(z)\left\|f e^{-\phi}\right\|_{L^{2}(\mathbb{C})}
$$

and the norm of $M^{R}$ goes to 0 when $R \rightarrow \infty$. So we have that $M=M_{\delta}+M_{\delta}^{R}+M^{R}$ is compact because the norm of $M_{\delta}+M^{R}$ can be made arbitrarily small and $M_{\delta}^{R}$ is compact.

Remark. The proof of Theorem 1.3 shows an interesting feature. The compactness of the canonical solution operator to $\bar{\partial}$ follows if the restriction of the operator to $(0,1)$-forms with holmorphic coefficients is compact. A similar situation appears in [FS98], where compactness of the $\bar{\partial}$-Neumann operator for convex domains in $\mathbb{C}^{n}$ is characterized. The obstruction to compactness also happens already on the space of $(0,1)$-forms with holomorphic coeffcients, see for instance [Kra88].

Proposition 1.4 We will use again the Marcinkiewicz interpolation theorem. Because of the decay of $C(z, \zeta)$ we have for $f e^{-\phi} \rho \in L^{p}(\mathbb{C})$ that

$$
u(z)=\int_{\mathbb{C}} C(z, \zeta) f(\zeta) e^{\phi(z)-\phi(\zeta)} d m(\zeta),
$$

is a well defined function. Now the estimates on the kernel $C(z, \zeta)$,

$$
\int_{D(z)} \frac{d m(\zeta)}{|z-\zeta|} \lesssim \rho(z), \quad \text { and } \quad \int_{\mathbb{C}} \frac{d m(\zeta)}{\rho(\zeta) \exp d_{\phi}(z, \zeta)^{\epsilon}} \lesssim \rho(z)
$$

yield $\left\|u e^{-\phi}\right\|_{L^{\infty}(\mathbb{C})} \lesssim\left\|f e^{-\phi} \rho\right\|_{L^{\infty}(\mathbb{C})}$ and $\left\|u e^{-\phi}\right\|_{L^{1}(\mathbb{C})} \lesssim\left\|f e^{-\phi} \rho\right\|_{L^{1}(\mathbb{C})}$. The rest of the proof is similar to the proof of Theorem 1.3 .

Assume now that the operator is compact. Let $\left\{z_{j}\right\}$ be a sequence of complex numbers such that the disks $D\left(z_{j}\right)$ are pairwise disjoint. If

$$
f_{j}(z)=\frac{k_{z_{j}}(z)}{\rho\left(z_{j}\right)} d \bar{z}, \quad u_{j}(z)=\overline{\left(z-z_{j}\right)} \frac{k_{z_{j}}(z)}{\rho\left(z_{j}\right)},
$$

one has $\bar{\partial} u_{j}=f_{j}$ and

$$
\int_{\mathbb{C}}\left|f_{j}(z)\right|^{2} e^{-2 \phi(z)} \rho(z) d m(z) \lesssim 1
$$

and one can extract a converging subsequence of $\left\{u_{j}\right\}$. But as before, from

$$
\left\|u_{j}-u_{k}\right\|^{2}=\left\|u_{j}\right\|^{2}+\left\|u_{k}\right\|^{2}+2 \operatorname{Re}\left\langle u_{j}, u_{k}\right\rangle \rightarrow 0
$$

we get a contradiccion because $\left\|u_{j}\right\| \sim 1$ and $\left|\left\langle u_{j}, u_{k}\right\rangle\right| \rightarrow 0$ for a fixed $k$ when $j \rightarrow \infty$. 


\section{REFERENCES}

[Ber01] B. Berndtsson, Weighted estimates for the $\bar{\partial}$-equation. Complex analysis and geometry (Columbus, OH,1999), 43.57, Ohio State Univ. Math. Res. Inst. Plub., 9, de Gruyter, Berlin, 2001.

[BO97] J. Bruna, J. Ortega-Cerdà, On $L^{p}$-solutions of the Laplace equation and zeros of holomorphic functions, Ann. Scuola Norm. Sup. Pisa Cl. Sci. (4) 24, no. 3, 571-591, 1997.

[Chr91] M. Christ, On the $\bar{\partial}$ equation in weighted $L^{2}$ norms in $\mathbb{C}$, J. Geom. Anal. 1, no. 3, 193-230, 1991.

[Del98] H. Delin, Pointwise estimates for the weighted Bergman projection kernel in $\mathbb{C}^{n}$, using a weighted $L^{2}$ estimate for the $\bar{\partial}$ equation, Ann. Inst. Fourier (Grenoble) 48, no. 4, 967-997, 1998.

[FS98] S. Fu, E. J. Straube, Compactness in the $\bar{\partial}-$ Neumann problem on convex domains, J. Funct. Anal. 159, no. 2, 629-641, 1998.

[FS01] S. Fu, E. J. Straube, Compactness in the $\bar{\partial}-$ Neumann problem, Complex Analysis and Geometry, J.McNeal ed., Ohio State Math. Res. Inst. Publ. 9, 141-160, 2001.

[Has06] F. Haslinger, Magnetic Schrödinger operators and the $\bar{\partial}$-equation, J. Math. Kyoto Univ. 46, no. 2 , 249-257, 2006.

[HH07] F. Haslinger, B. Helffer, Compactness of the solution operator to $\bar{\partial}$ in weighted $L^{2}$-spaces, J. Funct. Anal. 243, no. 2, 679-697, 2007.

[Ker72] N. Kerzman, The Bergman kernel function. Differentiability at the boundary, Math. Ann., 195, 149$158,1972$.

[Kra88] S. Krantz, Compactness of the $\bar{\partial}-$ Neumann operator, Proc. Amer. Math. Soc. 103, no. 4, 1136-1138, 1988

[Lin01] N. Lindholm, Sampling in weighted $L^{p}$ spaces of entire functions in $\mathbb{C}^{n}$ and estimates of the Bergman kernel, J. Funct. Anal., 182, 390-426, 2001.

[MMO03] N. Marco, X. Massaneda, J. Ortega-Cerdà, Interpolating and sampling sequences for entire functions, Geom. Funct. Anal. 13, no. 4, 862-914, 2003.

Department of Mathematical Sciences, Norwegian University of Science and TeChnOlogy, N-7491 TRONDHEIM, NORWAY

E-mail address: jordi.marzo@math.ntnu.no

Departament de Matemàtica Applicada i AnÀlisi, Universitat de Barcelona, Gran Via 585, 08007-BARCELONA, SPAIN

E-mail address: jortega@ub.edu 